\title{
On the numerical simulation of turbulent pipe flow pattern using Comsol Multiphysics
}

\author{
O. J. Adekanle ${ }^{* 1}$, A.A Adetoyinbo ${ }^{1}$, L. O. Mustapha ${ }^{2}$ and O. T. Kolebaje ${ }^{1}$ \\ ${ }^{1}$ Department of Physics, University of Ibadan, Ibadan, Nigeria \\ ${ }^{2}$ Department of Physics with Electronics, Lagos State Polytechnic, Ikorodu, Nigeria \\ *Corresponding authorE-mail:ojadekanle@yahoo.com or muslaty2k@gmail.com
}

\begin{abstract}
In this paper, two dimensional pipe of length $0.8 \mathrm{~m}$ and diameter $0.3 \mathrm{~m}, 0.4 \mathrm{~m}$, and $0.45 \mathrm{~m}$ were simulated respectively using Comsol Multiphysics 4.0 software. The governing equations and the k. $\varepsilon$ model which accounts for the turbulent kinetic energy $\mathrm{k}$ and turbulent dissipation rate $\varepsilon$ were solved using the simulator. The behaviors of the fluid were investigated at radius of $0.23 \mathrm{~m}, 0.33 \mathrm{~m}$, and $0.38 \mathrm{~m}$ respectively near the pipe wall to study its possible impact on the pipe wall. Changing in flow pattern with increasing velocity was observed which may cause a phenomenon such as heat transfer and consequently increase the mixing rate of dissolved substance. However, the results showed there is an indication that the inner wall of the pipes coated with thin film that reduces corrosion gradually decreases down the pipes due to the impact of the turbulent kinetic energy and turbulent dissipation rate on the pipe walls.
\end{abstract}

Keywords: Comsol Multiphysics, Flow in pipes, k.\& model, Turbulent flow.

\section{Introduction}

Turbulence, the chaotic and apparently unpredictable state of a fluid, is one of the most challenging problems in fluid dynamics. But from physical point of view there are many common properties of turbulent flows which can be recognized: time and space irregularity, strong mixing, high diffusivity and high vorticity, viscous dissipation, flow structures with continuous spectra of length and time scales, large Reynolds numbers and a three dimensional character, among others [1]. The smoke of a fire, the wake generated by a moving ship and the flow near a flying aeroplane are examples of turbulent flows. Since turbulence strongly increases the mixing and friction in flows, it is an issue of great practical significance in technology [2]. Numerous scientists have put much effort into the observation, description and understanding of turbulent flows. More than a century ago [3] demonstrated that a flow changes from an orderly to a turbulent state when a certain parameter, now called the Reynolds number, exceeds a critical value. Furthermore, an hierarchy of eddies (whirls) from large to small scales exists in turbulent flows, in which larger eddies transfer energy to smaller eddies, whereas the smallest eddies are dissipated by molecular viscosity.

Currently, several approaches to study turbulent flows exist: analytical theory, physical experiment and numerical simulation [4]. The complexity of the problem strongly slows down progress in the analytical approach. Experimental research has been conducted for many years and will remain of fundamental importance in this field. Closely connected with the increase of computational power in recent years, growing attention is paid to the numerical simulation of turbulence [5], which is also the approach followed in this thesis. This approach is advantageous over experiments when many flow quantities at a single instance or quantities which are difficult to measure are needed [6].

However, the present study focused on the turbulent flow pattern and velocity distribution along pipes whereas the specific objective is to study the effect of turbulent kinetic energy and turbulent dissipation rate on the pipe wall using k. $\varepsilon$ model. The k. $\varepsilon$ model is one of the most used turbulence models for industrial applications. The model introduces two additional transport equations and two dependent variables; the turbulence kinetic energy, $\mathrm{k}$ and the turbulence dissipation rate, $\varepsilon$. The standard model is very suitable for completely turbulent. 


\section{Materials and methods}

\subsection{Basic theory of fluid flow}

Fluid flow is divided into two regimes namely; the laminar regime and the turbulent regime. However, when a flow ceases to be laminar it just does not become turbulent but goes through a process of becoming turbulent, this process is called transition to turbulence. The different regimes of fluid flow are function of the Reynolds number. Reynolds number $(\mathrm{Re})$ is a dimensionless number that gives a measure of the ratio of inertial forces to viscous forces and consequently quantifies the relative importance of these two types of forces for given flow conditions. Mathematically, it can be expressed as

$R e=\frac{\rho v D}{\mu}$

Where

$\mathrm{v}$ is the mean velocity of the fluid $(\mathrm{m} / \mathrm{s})$

$\mathrm{D}$ is the diameter of the pipe considered (m)

$\mu$ is the dynamic viscosity of the fluid ( $\mathrm{Ns} / \mathrm{m}^{2}$, centipoise or pas)

$\rho$ is the density of the fluid $\left(\mathrm{kg} / \mathrm{m}^{3}\right)$

The flow is Laminar for $\operatorname{Re}<2000$, transitional between $2000<\operatorname{Re}<4000$, and turbulent for $\operatorname{Re}>4000$.

The flow field is assumed to satisfy the continuity and momentum equation for an incompressible fluid. These can be expressed as shown below

Continuity equation

$\nabla . U=0$

Momentum equation

$\frac{\partial u}{\partial t}+u \cdot \nabla u=-\nabla p+\frac{1}{R e} \nabla^{2} u$

(2) can be splitted further into

$\frac{\partial u}{\partial t}+u \frac{\partial u}{\partial x}+v \frac{\partial u}{\partial y}=-\frac{\partial p}{\partial x}+\frac{1}{R e}\left(\frac{\partial^{2} u}{\partial x^{2}}+\frac{\partial^{2} u}{\partial y^{2}}\right)$

$\frac{\partial v}{\partial t}+u \frac{\partial v}{\partial x}+v \frac{\partial v}{\partial y}=-\frac{\partial p}{\partial y}+\frac{1}{R e}\left(\frac{\partial^{2} v}{\partial x^{2}}+\frac{\partial^{2} v}{\partial y^{2}}\right)$

Subtracting the Derivative of (3a) with respect to $y$ from the derivative of (3b) with respect to $\mathrm{x}$ and defining vorticity $\mathrm{w}$ as

$w=\frac{\partial v}{\partial x}-\frac{\partial u}{\partial y}$

Result to

$\frac{\partial w}{\partial t}+u \frac{\partial w}{\partial x}+v \frac{\partial w}{\partial y}=\frac{1}{R e}\left(\frac{\partial^{2} w}{\partial x^{2}}+\frac{\partial^{2} w}{\partial x^{2}}\right)$

Applying Stream Function

Let $\mathrm{u}=\frac{\partial \psi}{\partial y} \quad \mathrm{v}=-\frac{\partial \psi}{\partial x}$

Substituting (5) into (4)

$\frac{\partial w}{\partial t}+\frac{\partial \psi}{\partial y} \frac{\partial w}{\partial x}-\frac{\partial \psi}{\partial x} \frac{\partial w}{\partial y}=\frac{1}{R e}\left(\frac{\partial^{2} w}{\partial x^{2}}+\frac{\partial^{2} w}{\partial y^{2}}\right)$

$\rightarrow \frac{\partial w}{\partial t}=-\frac{\partial \psi}{\partial y} \frac{\partial w}{\partial x}+\frac{\partial \psi}{\partial x} \frac{\partial w}{\partial y}+\frac{1}{R e}\left(\frac{\partial^{2} w}{\partial x^{2}}+\frac{\partial w}{\partial y^{2}}\right)$

Equation (6) is the Navier-Stoke equation in vorticity stream function form.

\subsection{Method}

Two dimensional pipe of length $0.8 \mathrm{~m}$ and diameter $0.3 \mathrm{~m}, 0.4 \mathrm{~m}$, and $0.45 \mathrm{~m}$ were simulated respectively using Comsol Multiphysics 4.0 software. The governing equations and the k. $\varepsilon$ model were solved using the simulator. The k. $\varepsilon$ model is a two equations model meaning that it takes into account two extra transport equations in order to account for the turbulent properties of the flow. Equation 7 takes into account the turbulent kinetic energy k, and determines the energy of the turbulence. Equation 8 is called the turbulent dissipation rate $\varepsilon$, and it is the variable that determines the scale of the turbulence.

$\frac{\partial(\rho k)}{\partial t}+\operatorname{div}(\rho k U)=\operatorname{div}\left[\frac{\mu t}{\sigma k} \operatorname{grad} k\right]+2 \mu_{t} E_{i j} E_{i j}-\rho \varepsilon$
$\frac{\partial(\rho \varepsilon)}{\partial t}+\operatorname{div}(\rho \varepsilon U)=\operatorname{div}\left[\frac{\mu t}{\sigma \varepsilon} \operatorname{grad} \varepsilon\right]+C_{1 \varepsilon} \frac{\varepsilon}{k} 2 \mu_{t} E_{i j} E_{i j}-C_{2 \varepsilon} \rho \frac{\varepsilon^{2}}{k}$

The constant for the k. $\varepsilon$ model [7] is shown in Table 1below 
Table 1: Constant for the k.e model.

\begin{tabular}{ccccc}
\hline$C_{\mu}$ & $C_{1}$ & $C_{2}$ & $\sigma_{k}$ & $\sigma_{\varepsilon}$ \\
\hline 0.09 & 1.44 & 1.92 & 1.0 & 1.3 \\
\hline
\end{tabular}

Table 2: Shows the characteristics of the fluid used.

\begin{tabular}{cc}
\hline Type of fluid & Kerosene \\
\hline Density & $790\left(\mathrm{Kg} / \mathrm{m}^{3}\right)$ \\
Viscosity & $0.00164 \mathrm{pas}$ \\
Input velocity & $200,300,400(\mathrm{~m} / \mathrm{s})$ \\
\hline
\end{tabular}

The k.e turbulence model relies on several assumptions, the most important of which are that the Reynolds number is high enough and that the turbulence is in equilibrium in boundary layers, which means that the production equal dissipation. The assumptions limit the accuracy of the model; since this is not always true [8].

Since it was desired to model turbulence, the wall functions condition was used. After all the conditions were set, the model meshed using physics controlled mesh and then solved. Three simulations were performed for pipe diameter of $0.3 \mathrm{~m}, 0.4 \mathrm{~m}$ and $0.45 \mathrm{~m}$ respectively. The behaviors of the fluid were investigated at radius of $0.23 \mathrm{~m}, 0.33 \mathrm{~m}$, and $0.38 \mathrm{~m}$ to see the possible effect of turbulence on the pipe wall.

\section{Results and discussion}

The graphs below showed the results obtained for velocity distribution, turbulent kinetic energy and turbulent dissipation rate.

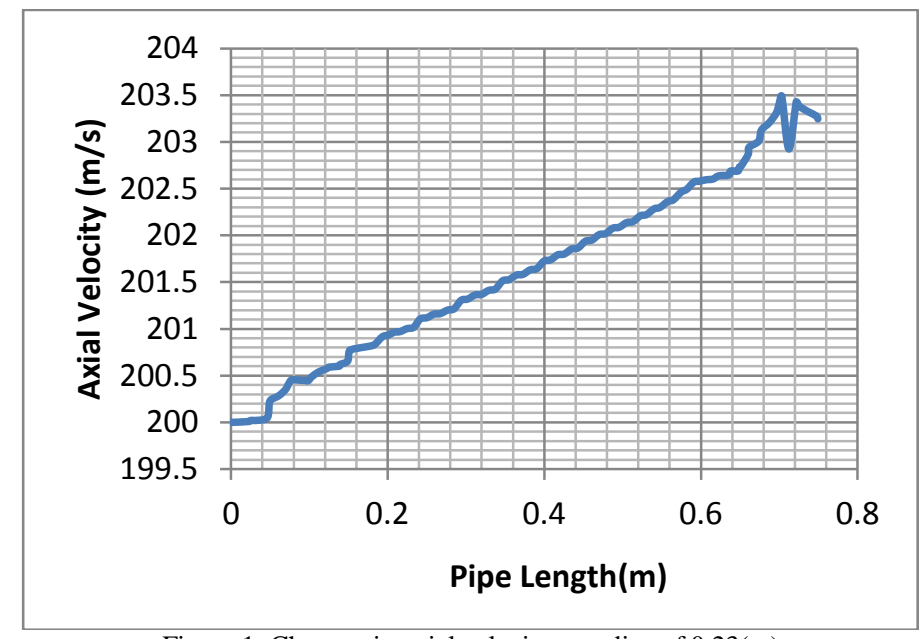

Figure 1: Changes in axial velocity at radius of $0.23(\mathrm{~m})$

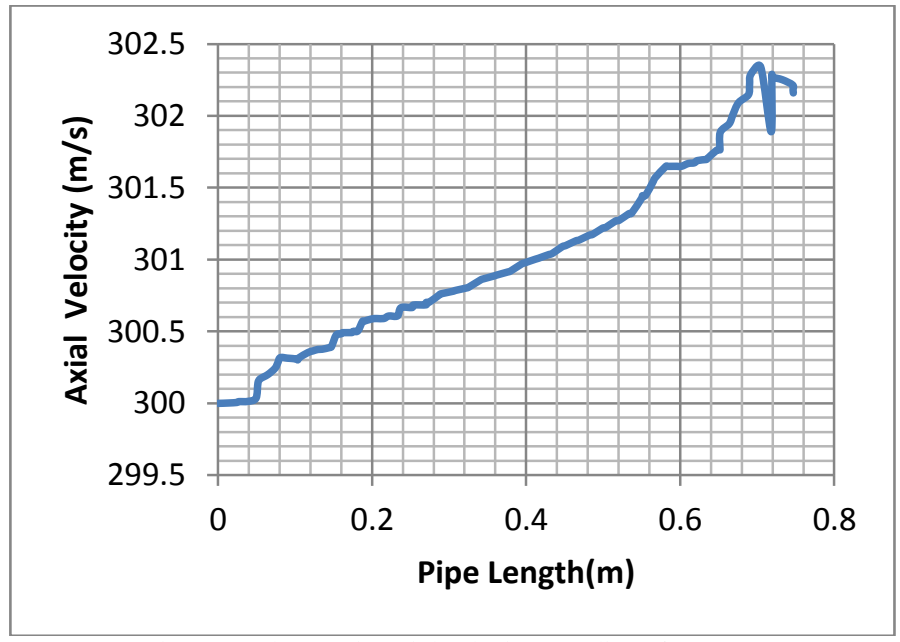

Figure 2: Changes in axial velocity at radius of $0.33(\mathrm{~m})$ 


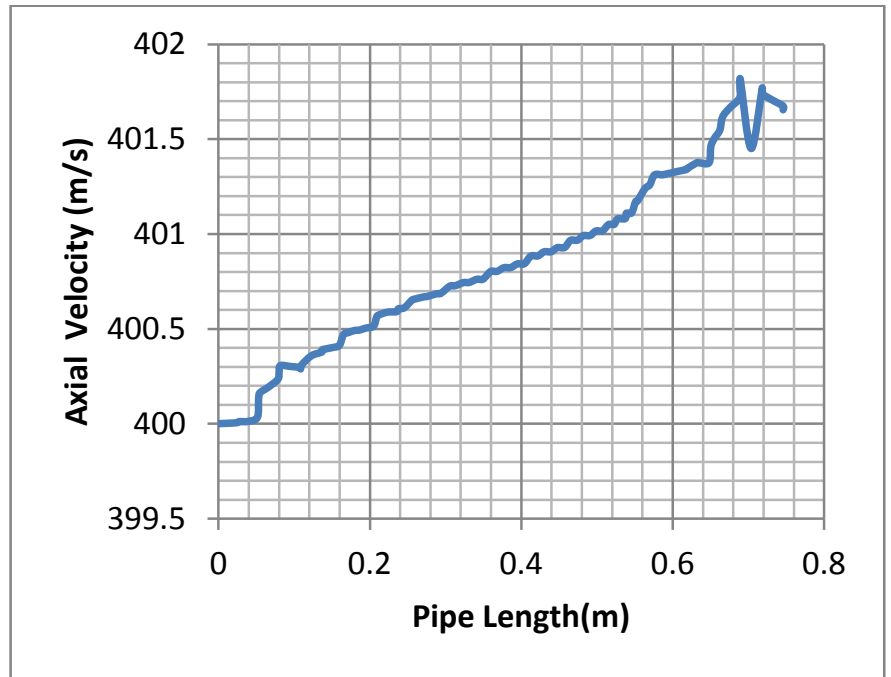

Figure 3: Changes axial velocity at radius of $0.38(\mathrm{~m})$

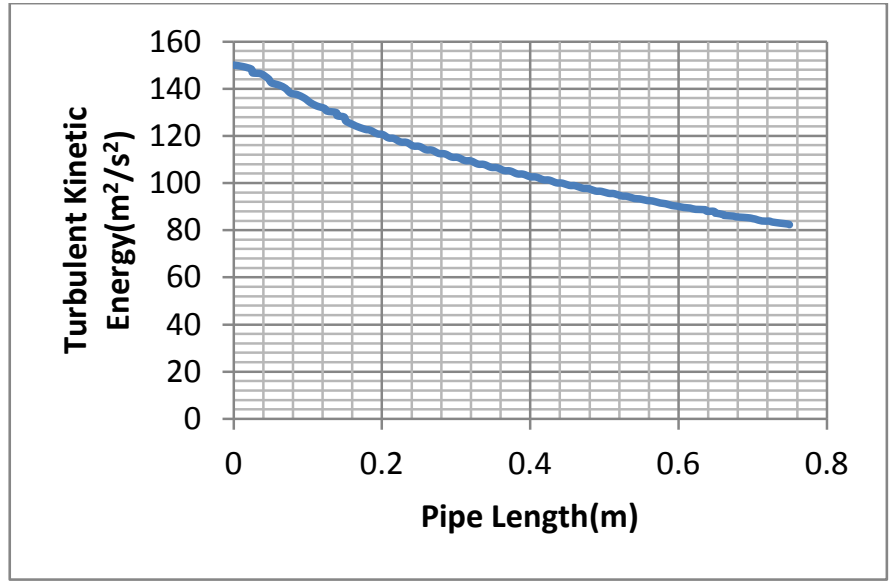

Figure 4: Turbulent kinetic energy at radius of $0.23(\mathrm{~m})$

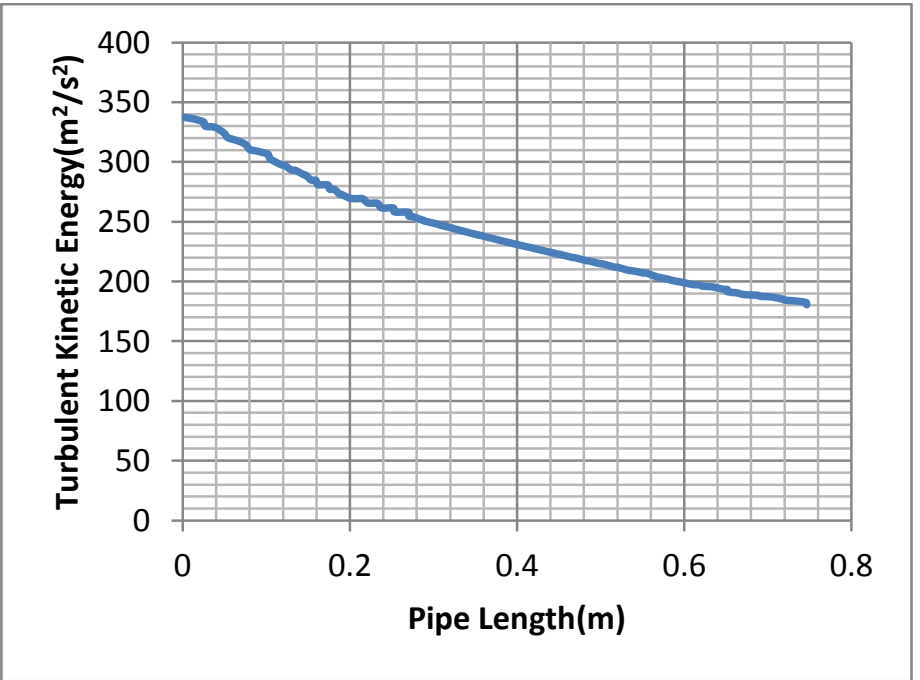

Figure 5: Turbulent kinetic energy at radius of $0.33(\mathrm{~m})$ 


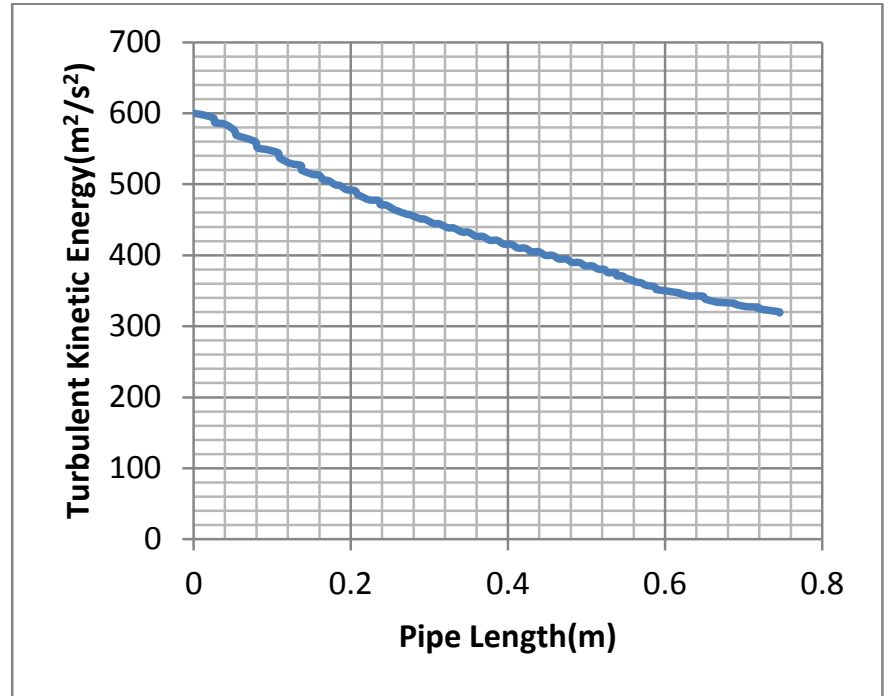

Figure 6: Turbulent kinetic energy at radius of $0.38(\mathrm{~m})$

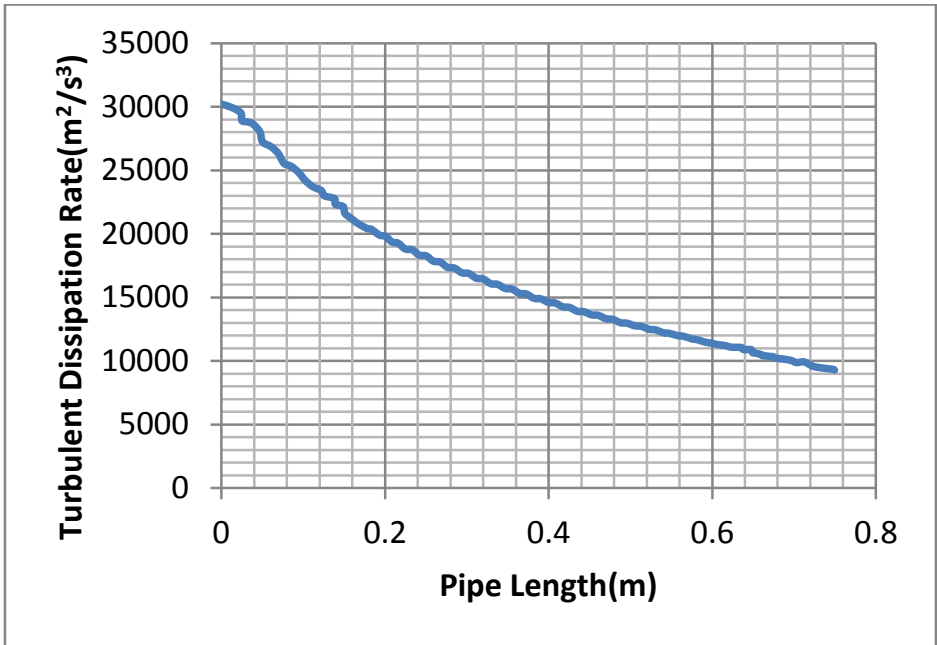

Figure 7: Turbulent dissipation rate at radius of $0.23(\mathrm{~m})$

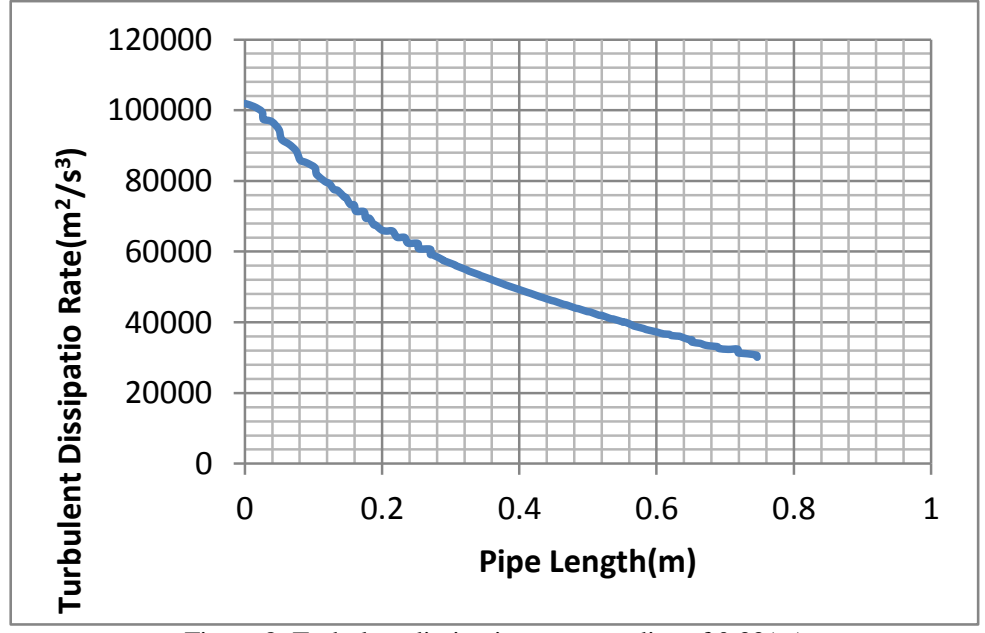

Figure 8: Turbulent dissipation rate at radius of $0.33(\mathrm{~m})$ 


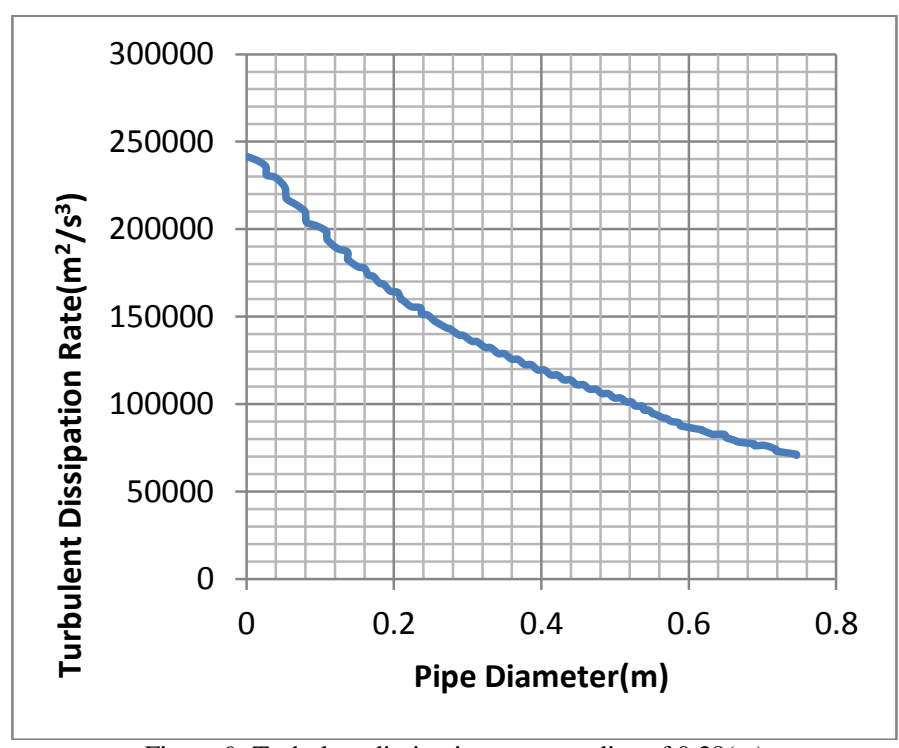

Figure 9: Turbulent dissipation rate at radius of $0.38(\mathrm{~m})$

The random, irregular nature and changes in flow pattern of the fluid with increasing velocity as revealed by figures $1-$ 3 is a distinguishing feature of turbulent flow. Changing in flow pattern may cause a phenomenon such as heat transfer and consequently increase the mixing rate of dissolved substance being transfer. However, the decreasing trends in figures $4-9$ is an indication that the inner wall of the pipes coated with thin film that reduces corrosion gradually decreases down the pipes due to the impact of the turbulent kinetic energy and turbulent dissipation rate on the pipe walls.

\section{Conclusion}

In many industries like oil and chemical ones, turbulence is very relevant hence, it is important to study its flow pattern and investigate its possible effect on pipe walls. Hence, it is revealed in the course of the research that the flow pattern of the fluid changes with increasing velocity. However, the effect of the turbulent kinetic energy and turbulent dissipation rate on the pipe wall also decreases down the pipe. The present investigation provides valuable insight into the dynamics of flow in fluid transport pipelines. Further investigation in dynamics of flow, velocity distribution, turbulent kinetic energy and dissipation rate which is influence on heat transfer, corrosion rate etc, needs to be studied in more detail, especially for three dimensional flows.

\section{References}

[1] Vreman, A. W., Sandham, N. D., and Luo, K. H., Compressible mixing layer growth rate and turbulence characteristics, J. Fluid Mech., vol. 10, no. 5, (1995), pp. $123-130$.

[2] H. Tennekes and J. L. Lumley, A First Course in Turbulence, The MIT Press, (1972).

[3] Reynolds, O., An experimental investigation of the circumstances which determine whether the motion of water shall be direct and sinuous, and the law of resistance in parallel channels, Phil. Trans. Roy. Soc., (1883), pp. 51-105.

[4] Vreman, B., Geurts, B., and Kuerten, H., Comparison of subgrid-models in Large Eddy Simulation of the temporal mixing layer, Memorandum of University of Twente, vol. 1243, (1994).

[5] Simone, A., and Cambon, C., Rapid distortion and direct simulation approach to compressibility in turbulent shear flow, Proceedings Turbulent Shear Flows, vol. 10, The Pennsylvania State University, (1995).

[6] Schumann, U., Direct and large eddy simulation of turbulence, BGT press, (1991).

[7] Launder, B. E., and Sharma, B. I., Application of the Energy Dissipation Model of Turbulence to the Calculation of Flow Near a Spinning Disc, Letters in Heat and Mass Transfer, vol. 1, no. 2, (1974), pp. 131-138.

[8] Michael A. T., Simulation of Turbulent Pipe Flow, Durfly press, (1999). 